\title{
Some Observations on Carbon Nanotubes Susceptibility to Cell Phagocytosis
}

\author{
Aneta Fraczek-Szczypta, ${ }^{1}$ Elzbieta Menaszek, ${ }^{1,2}$ and Stanislaw Blazewicz ${ }^{1}$ \\ ${ }^{1}$ Department of Biomaterials, Faculty of Materials Science and Ceramics, AGH-University of Science and Technology, \\ al Mickiewicza 30, 30-059 Krakow, Poland \\ ${ }^{2}$ Department of Cytobiology, Collegium Medicum, Jagiellonian University, Medyczna 9, 30-068 Krakow, Poland
}

Correspondence should be addressed to Aneta Fraczek-Szczypta, afraczek@agh.edu.pl

Received 30 September 2010; Accepted 30 December 2010

Academic Editor: Xiaojun Yu

Copyright (c) 2011 Aneta Fraczek-Szczypta et al. This is an open access article distributed under the Creative Commons Attribution License, which permits unrestricted use, distribution, and reproduction in any medium, provided the original work is properly cited.

The aim of this study was to assess the influence of different types of carbon nanotubes (CNTs) on cell phagocytosis. Three kinds of carbon nanotubes: single-walled carbon nanohorns (SWCNHs), multiwalled carbon nanotubes (MWCNTs), and ultralong single-walled carbon nanotubes (ULSWCNTs) before and after additional chemical functionalization were seeded with macrophage cell culture. Prior to biological testing, the CNTs were subjected to dispersion process with the use of phosphate buffered solution (PBS) and PBS containing surfactant (Tween 20) or dimethyl sulfoxide (DMSO). The results indicate that the cells interaction with an individual nanotube is entirely different as compared to CNTs in the form of aggregate. The presence of the surfactant favors the CNTs dispersion in culture media and facilitates phagocytosis process, while it has disadvantageous influence on cells morphology. The cells phagocytosis is a more effective for MWCNTs and SWCNHs after their chemical functionalization. Moreover, these nanotubes were well dispersed in culture media without using DMSO or surfactant. The functionalized carbon nanotubes were easily dispersed in pure PBS and seeded with cells.

\section{Introduction}

Carbon nanotubes (CNTs) are being produced in increasingly large quantities for many technical and medical applications due to their novel properties, such as enhanced thermal, electronic, mechanical, and biological properties. In biological systems, they have been investigated as drug delivery vehicles, targeted cancer therapies, tissue scaffolds, and biosensors [1-3]. CNTs due to their relative large lengthto-diameter aspect ratio, with a very large specific surface are suitable for highly sensitive molecular detection and recognition. Consequently, a large fraction of the CNT surface can be modified with functional groups of various complexities, which would modulate its in vivo and in vitro behaviour [4].

However, opinions about the biocompatibility of CNTs in vitro and in vivo environments are not unequivocal. Some authors indicate that CNTs are biocompatible in contact with cells and tissue, that is, they stimulate osteoblast and nerve cells to grow and proliferate and induce muscle and blood vessels to regenerate [5-7]. Contrary to these outcomes, many critical results point to the cytotoxicity of CNTs. The toxicity of CNTs is a prime concern, and several groups point to their similarity to asbestos fibres [8]. Other scientists indicate that CNTs may lead to dermal toxicity due to accelerated oxidative stress in the skin and pulmonary toxicity through induced lung lesions characterized by the presence of granulomas $[9,10]$.

The question arises why the opinions about the biocompatibility of carbon nanotubes are so different?

Many scientists explain that CNTs toxicity in both in vivo and in vitro studies has been attributed to various factors, for instance, length, type of functionalization, their concentration, duration and method of exposure, catalyst impurity, agglomeration, and even the dispersants used to dissolve the nanotubes $[4,11-16]$. However, most aspects of CNT toxicity remain still not properly recognized. One of the important aspects is the transport of CNTs across the 


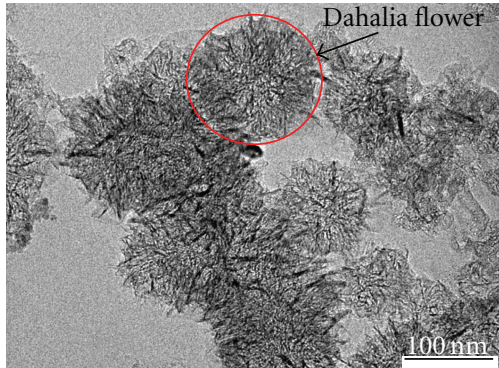

(a)

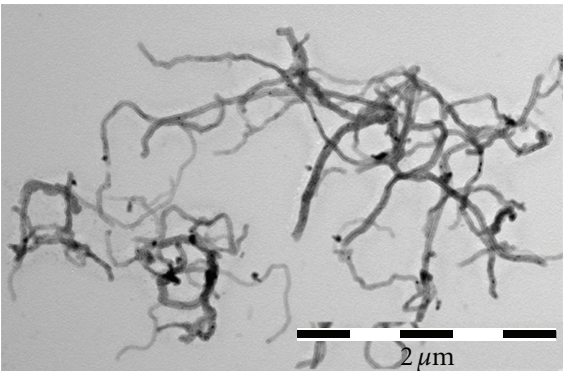

(b)

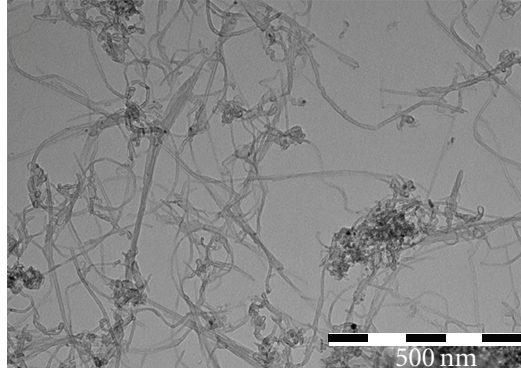

(c)

FIgURE 1: TEM microphotography of SWCNH (a), MWCNT (b), and ULSWCNT (c) before chemical functionalization.

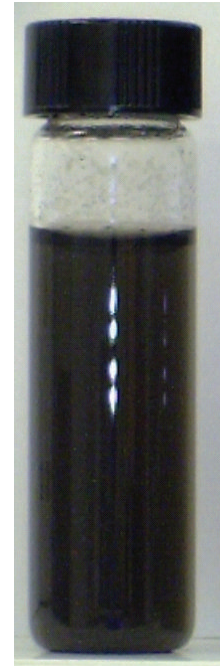

(a)

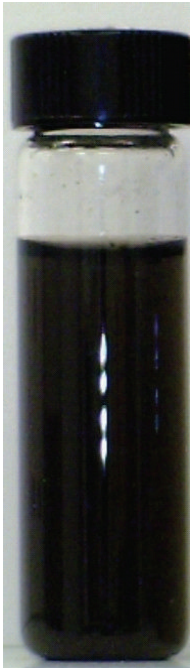

(b)
FIgURE 2: MWCNT in 10\% DMSO just after dispersion (a) and $48 \mathrm{~h}$ later (b).

cell membrane. Two potential transport mechanisms of CNT have been considered: endocytosis/phagocytosis and energyindependent passive process $[4,17,18]$.

The aim of this study is to examine the effect of dispersion degree of CNTs on cell phagocytosis process. The CNTs dispersion depends upon a number of factors, such as the type of CNTs, their geometry, the presence of surface chemical state as well as the type of surfactants. These factors are qualitatively analyzed in view of differently prepared CNTs to their susceptibility to cells response.

\section{Materials and Methods}

Five kinds of pristine and functionalized carbon nanotubes (CNTs) were used in this work. The pristine CNTs were provided by NanoAmor USA and NanoCraft Inc USA. The pristine carbon nanotubes used in this experiment were denoted, as follows (Figure 1):

(i) SWCNH_-single wall carbon nanohorns,

(ii) MWCNT-multi wall carbon nanotubes,

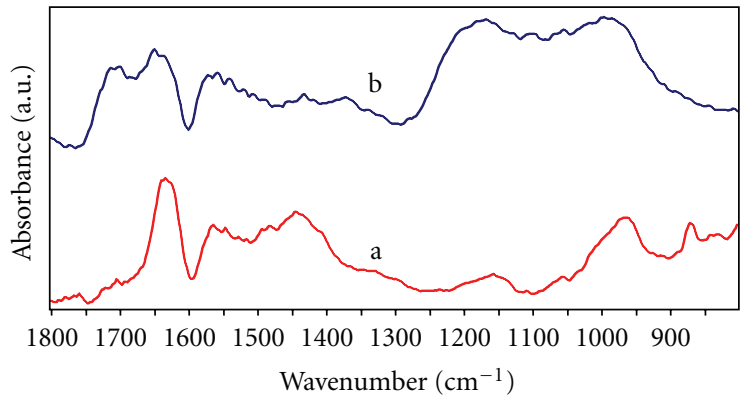

Figure 3: IR spectra of MWCNT (a) and MWCNT-F (b).

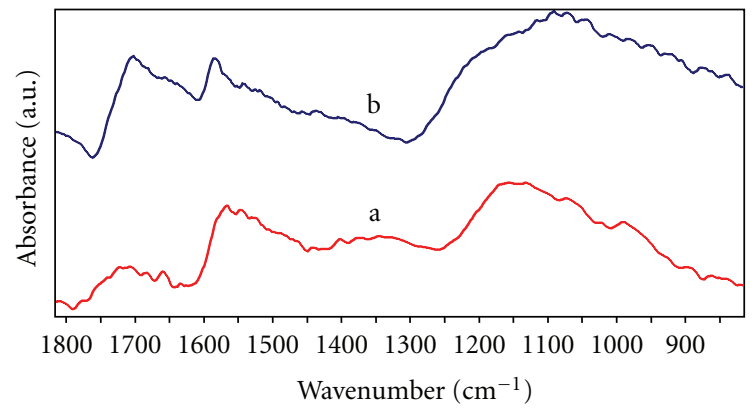

FIgURE 4: IR spectra of SWCNH (a) and SWCNH-F (b).

(iii) ULSWCNT-ultra-long-single-wall carbon nanotubes.

The diameters of SWCNHs ranged from 2 to $3 \mathrm{~nm}$ and length from 30 to $50 \mathrm{~nm}$, with a $19^{\circ}$ closed-end called car bon nanohorns. Carbon nanohorns belong to the carbon nanotubes family. The tip of this tube is closed by a conical cap with a cone angle of $19^{\circ}$. The individual carb on nanohorns have a tendency to aggregate together and form dahlia-flower-like structured particles (Figure 1(a)). SWCNH aggregates have been regarded as potentially good drug carriers, which possess some advantages over other drug carriers $[2,19]$.

MWCNTs had diameters in the range of $10-30 \mathrm{~nm}$ and were 1-2 $\mu \mathrm{m}$ long, whereas ULSWCNTs had diameters from 0.7 to $2 \mathrm{~nm}$ and length from $15-30 \mu \mathrm{m}$. 
TABLE 1: Purity of carbon nanotubes before and after chemical oxidative treatment.

\begin{tabular}{|c|c|c|c|c|c|}
\hline \multirow{2}{*}{ Chemical oxidation } & \multirow{2}{*}{ Samples } & \multicolumn{4}{|c|}{ Concentration (wt.\%) } \\
\hline & & $\mathrm{Fe}$ & Co & $\mathrm{Al}$ & $\mathrm{Ni}$ \\
\hline Before & SWCNH & 1.8 & 0.007 & 0.5 & 0.2 \\
\hline After & SWCNH-F & 0.05 & 0.0001 & 0.04 & 0.003 \\
\hline Before & MWCNT & 0.3 & 0.006 & 0.06 & 1.2 \\
\hline After & MWCNT-F & 0.01 & 0.001 & 0.01 & 0.1 \\
\hline Before & ULSWCNT & 0.003 & 0.8 & 0.02 & 0.002 \\
\hline After & ULSWCNT-F & 0.0001 & 0.08 & 0.006 & 0.0003 \\
\hline
\end{tabular}

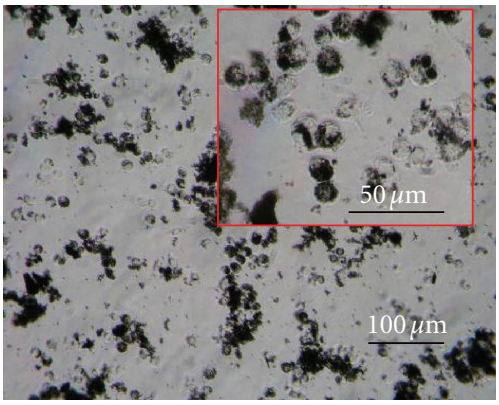

(a)

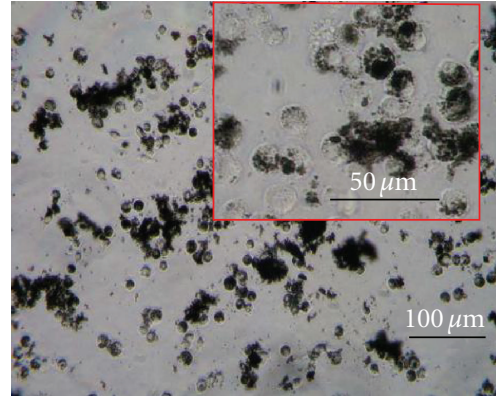

(b)

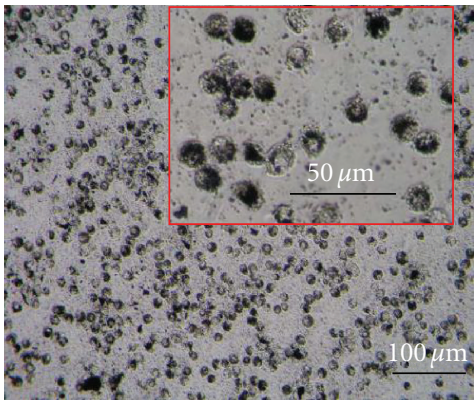

(c)

FIgURE 5: Macrophages in contact with SWCNH dispersed in PBS (a), in PBS + 1\% DMSO (b), and in PBS + 1\% Tween 20 (c).

A mixture of concentrated $\mathrm{H}_{2} \mathrm{SO}_{4}$ and $\mathrm{HNO}_{3}$ with volume ratio of $3: 1$ was made for functionalization of the CNTs. They were immersed into this mixture and kept four hours at temperatures between $60^{\circ} \mathrm{C}$ and $70^{\circ} \mathrm{C}$. The main aim of this process was the removal of metallic catalysts and chemical modification by introducing of carboxyl acid groups on the CNT surface. In this way, two types of samples were prepared

(i) SWCNH-F-SWCNHs after chemical purification in concentrated acid,

(ii) MWCNT-F-MWCNTs after chemical purification in concentrated acid.

CNTs were sterilized by the UV method for $0.5 \mathrm{~h}$. Before incubation with cells, each type of carbon nanotubes was sonicated for $1 \mathrm{~min}$ using a tip sonicator (PALMER INSTRUMENTS, Model: CP 130 PB) in three different solutions:

(i) PBS- phosphate buffered solution,

(ii) $\mathrm{PBS}+1 \%$ DMSO (DMSO-dimethyl sulfoxide),

(iii) $\mathrm{PBS}+1 \%$ Tween 20 ,

with a concentration of CNT $38 \mu \mathrm{g} / \mathrm{mL}$ (a safe concentration level of CNTs is to be around $40 \mu \mathrm{g} / \mathrm{mL}$ ) [20]. Dimethyl sulfoxide (DMSO) used in our experiments is an appropriate polar aprotic solvent that dissolves both polar and nonpolar compounds, miscible in a wide range of organic solvents as well as in water. In medicine, DMSO is predominantly used as a topical analgesic, a vehicle for topical application of pharmaceuticals, as an anti-inflammatory agent and as an antioxidant. Because DMSO enhances the rate of absorption of some compounds through organic tissues including skin, it can be used as a drug delivery system. This solvent has acceptable biological properties, and the suspensions prepared with the dispersed CNTs perform a long-lasting stability (Figure 2).

Tween 20 is a stable and a relative nontoxic detergent and emulsifier used in a number of pharmacological applications. It was used as a dispersion agent of carbon nanotubes in previous works [21, 22]. After sonication, all kinds of CNTs were observed in contact with RAW 264.7 for $24 \mathrm{~h}$.

The morphology of CNTs before functionalization was analyzed using transmission electron microscopy (TEM) (Tecnai G2 F20 (200 kV) and Joel). The degree of purification of CNTs was determined using inductively coupled plasma optical emission spectrometry (ICP-OES) (Multiwave 3000, Perkin Elmer Co.). Evaluation of the functionalization of carbon nanotubes was done using Fourier transform infrared spectroscopy (FTIR) (Bio-Rad FTS60V spectrometer). The transmission of FTIR spectra was registered in the range of $800-1800 \mathrm{~cm}^{-1}$ using $\mathrm{KBr}$ pellets. The phagocytosis of nanotubes by RAW 264.7 macrophages was observed using inverted microscope (Olympus CKX41, Germany).

The murine macrophage RAW 264.7 cell line (ATCC, GB) was used in this study. The cells were cultured in 75$\mathrm{cm}^{2}$ tissue culture flasks (Nunc, Denmark) in Dulbecco's modified Eagle's medium (DMEM; PAA, Austria) supplemented with antibiotics (penicillin G $100 \mathrm{U} / \mathrm{mL}$, streptomycin $10 \mu \mathrm{g} / \mathrm{mL}$ (Sigma-Aldrich, Germany)) and 10\% fetal bovine serum (PAA, Austria). The flasks of cultured cells were incubated at $37^{\circ} \mathrm{C}$ in humidified $95 \%$ air and $5 \%$ $\mathrm{CO}_{2}$. Cells were routinely passaged by harvesting using a cell scraper and replated in tissue culture flasks at a ratio of $1: 5$ 


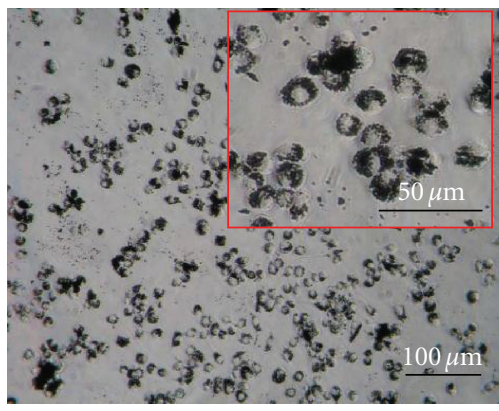

(a)

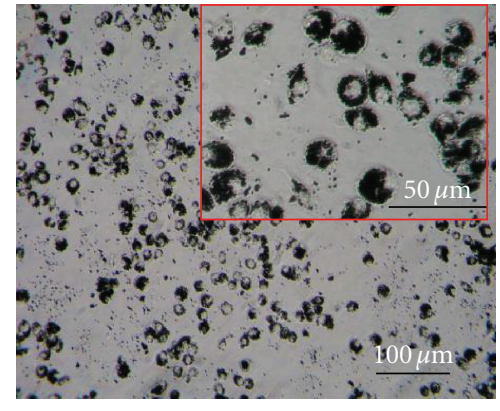

(b)

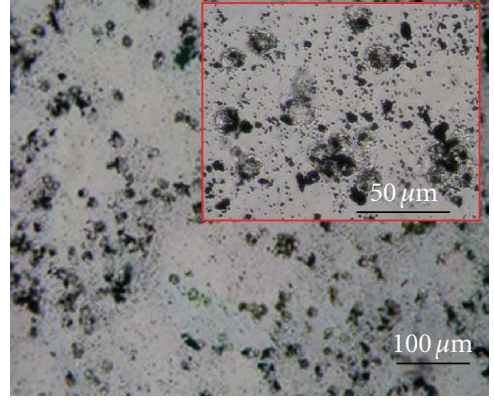

(c)

Figure 6: Macrophages in contact with SWCNH-F dispersed in PBS (a), in PBS + 1\% DMSO (b), and in PBS + 1\% Tween 20 (c).

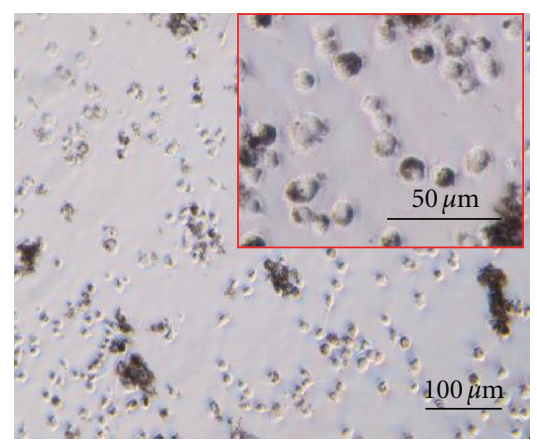

(a)

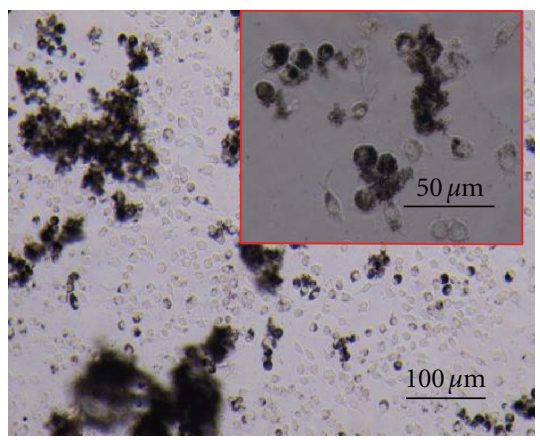

(b)

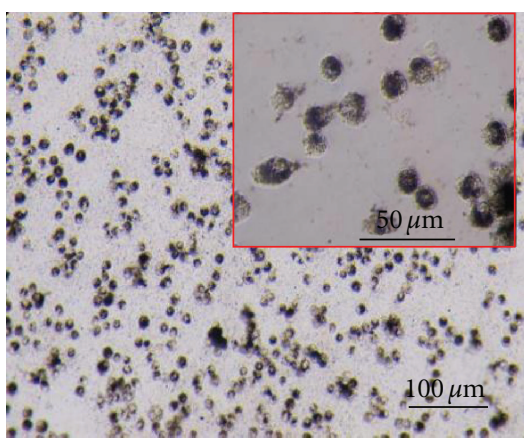

(c)

FIGURe 7: Macrophages in contact with MWCNT dispersed in PBS (a), in PBS + 1\% DMSO (b), and in PBS + 1\% Tween 20 (c).

for subculturing. Cells were passaged no more than 10 times before an experiment. In our experiment, cells were placed into 24-well dishes (Nunc, Denmark) at a population density of $5 \times 10^{4}$ cells $/ \mathrm{mL} /$ well and allowed to adhere for $2 \mathrm{~h}$. Next, $10 \mu \mathrm{L}$ of nanotubes dispersed in different media were added to cell culture. After $24 \mathrm{~h}$, the supernatant from above cells was collected for cytotoxic assay and cells in culture dishes were observed with inverted optical microscope.

\section{Results and Discussion}

Purity of carbon nanotubes was determined using ICP-OES. The results are gathered in Table 1.

ICP-OES analysis of the as-prepared SWCNH indicated mainly the presence of iron (Fe) catalyst $(1.8 \mathrm{wt} . \%)$ in this sample. In the case of the pristine MWCNT and ULSWCNT, the ICP-OES analysis showed mainly the presence of nickel (Ni) (1.2 wt.\%) and cobalt (Co) (0.8 wt.\%), respectively. Using this method, the effectiveness of metal catalysts removal from CNTs after the oxidation process in acid $\left(\mathrm{H}_{2} \mathrm{SO}_{4}: \mathrm{HNO}_{3}\right)$ was determined. The acid treatment of CNTs allowed to remove the metal catalyst residues in CNT samples, to open the end caps of the CNTs, and to leave them terminated with carboxylic groups [23-26]. The concentration of metal catalysts significantly decreases for all types of carbon nanotubes after chemical oxidation (Table 1). The conclusion drawn from this analysis is that the chemical oxidation method was useful for the purification of CNTs.
To clarify the influence of the acid mixture on the surface chemistry of MWCNT and SWCNH after the functionalization process, the FTIR investigation was carried out and the corresponding results are shown in Figures 3 and 4. The spectrum of the as-prepared CNTs shows the $\mathrm{C}-\mathrm{C}$ stretching bonds in the range of $1580-1650 \mathrm{~cm}^{-1}$ characteristic to the expected nanotube phonon modes [26]. The spectrum of the CNTs after oxidation presents two characteristic bands at $1710 \mathrm{~cm}^{-1}$ (for MWCNT-F) and at $1704 \mathrm{~cm}^{-1}$ (for SWCNHF) and a broad band in the range from 900 to $1220 \mathrm{~cm}^{-1}$ assigned to $\nu_{\mathrm{C}=\mathrm{O}}$ and $\nu_{\mathrm{C}-\mathrm{O}}$ carbonyl and carboxyl groups. These bands were not observed in the spectra of CNTs before the treatment. This implies that the oxygen-containing functional groups are introduced on the MWCNT and SWCNH surfaces during their oxidative treatment. These functional groups are usually found to be attached to the ends of the nanotubes or defects along their wall, due to the enhanced reactivity of these sites [26]. The characteristic bands of FTIR spectrum of other samples are gathered in Table 2. The presence of the chemical group on CNTs surface confirms the effectiveness of functionalization methods.

In order to identify the phagocytosis process, both pristine and functionalized CNTs were analyzed using macrophage cell line (RAW 264.7). The proper application of carbon nanotubes in a biological environment is directly connected with overcoming the agglomeration problem. Due to van der Waals interactions, carbon nanotubes have a strong tendency to orient themselves parallel to each other. 


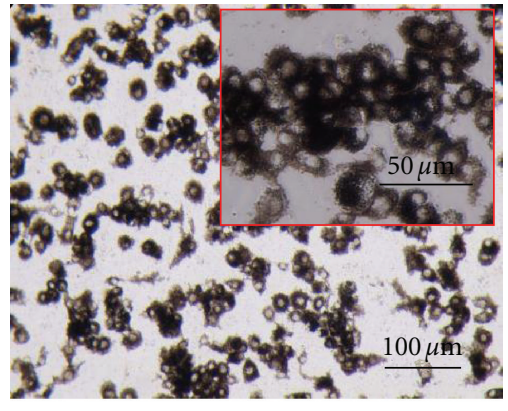

(a)

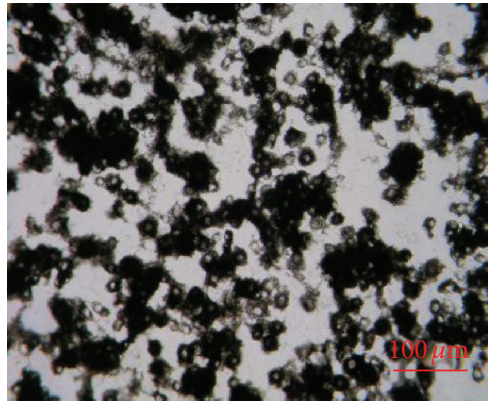

(b)

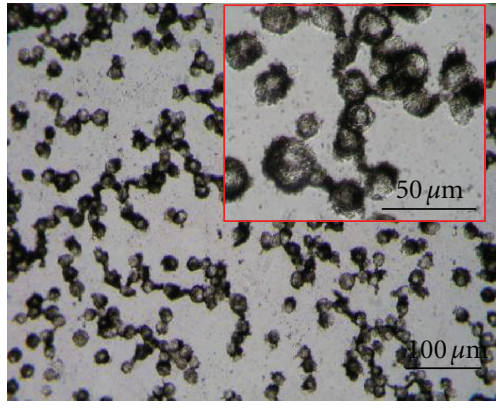

(c)

Figure 8: Macrophages in contact with MWCNT-F dispersed in PBS (a), in PBS + 1\% DMSO (b), and in PBS + 1\% Tween 20 (c).

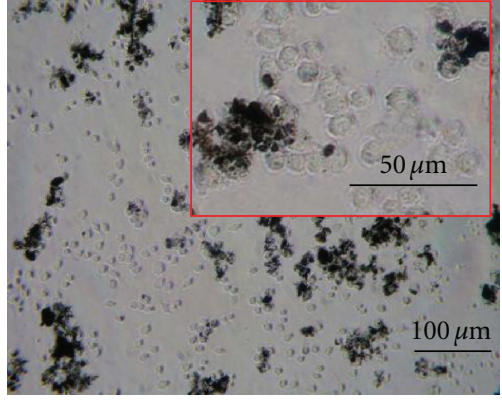

(a)

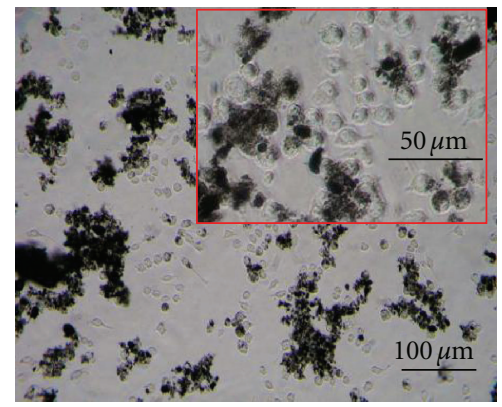

(b)

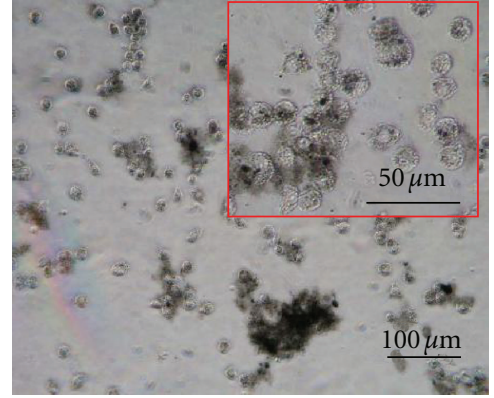

(c)

Figure 9: Macrophages in contact with ULSWCNT dispersed in PBS (a), in PBS + 1\% DMSO (b), and in PBS + 1\% Tween 20 (c).

TABLE 2: Results from FTIR spectroscopy of MWCNT and SWCNH after functionalization in acids.

\begin{tabular}{lcc}
\hline $\begin{array}{l}\text { MWCNT-F } \\
\text { Wavenumber }\left(\mathrm{cm}^{-1}\right)\end{array}$ & Chemical bond \\
\hline 1710 & 1704 & $\mathrm{C}=\mathrm{O}$ and C-O \\
$900-1220$ & $900-1220$ & \\
\hline
\end{tabular}

During such an interaction, the CNTs create agglomerates in the form of ropes and bundles with the binding energy accompanying this mechanism several hundreds of $\mathrm{meV} / \mu \mathrm{m}$ [27]. The properties of agglomerated carbon nanotubes are different than those observed for their single, separated forms [14-16]. Hence, the preliminary step before in vitro testing is their good dispersion in a medical environment. All kinds of CNTs were dispersed in the pure PBS or PBS containing $1 \%$ of DMSO or Tween 20 , at the concentration of $38 \mu \mathrm{g} / \mathrm{mL}$ and then introduced to DMEM. The dispersion was monitored using a digital camera (Camedia C5050Z, Olympus, Germany) after $24 \mathrm{~h}$. The sets of suspensions containing various CNTs prepared in different way are visualized in Figure 11.

The aggregates of all types of CNTs were dispersed using ultrasounds (results not shown). However, a part of carbon nanotubes after dispersion had a tendency to secondary aggregation (Figure 11). A complete sedimentation was observed for pristine CNTs in PBS solution and PBS with DMSO only (Figure $11(\mathrm{~A}, \mathrm{~B}, \mathrm{G}, \mathrm{H}, \mathrm{M}$, and $\mathrm{N})$ ). The Tween

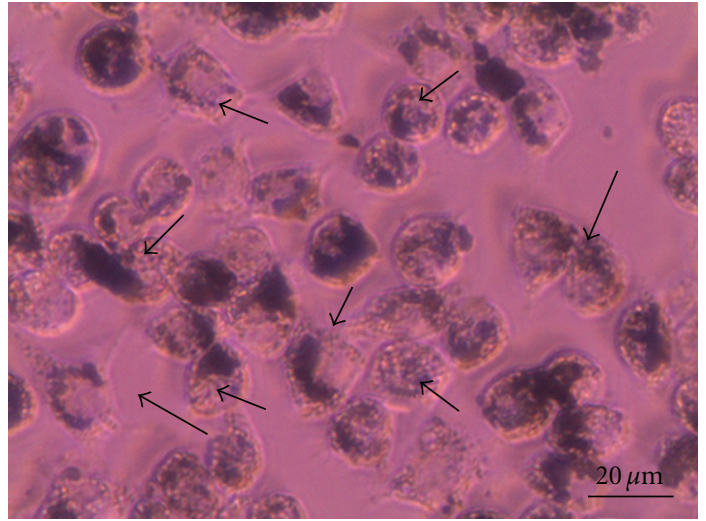

Figure 10: The macrophage cells after incubation with nanotubes dispersed in Tween 20 .

20 added to PBS improves the dispersity and stability of SWCNH and MWCNT in culture media (Figure 11(C and I)). A different situation was observed in the case of ULSWCNT, namely, the presence of Tween 20 did not inhibit the sedimentation process (Figure $11(\mathrm{O})$ ). The hydrophobic nature of the pristine CNTs has a decisive influence on the agglomeration process of CNT in PBS solution. Moreover, the presence of culture media ingredients (e.g., protein, hormones, vitamins, etc.) may have an impact on the sedimentation process. Due to nature of the Tween 20 surfactant, its presence in the PBS solution improves dispersion of 
SWCNH

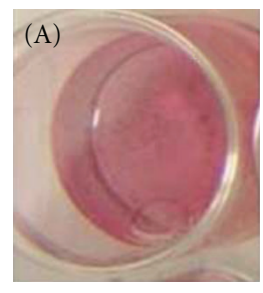

PBS

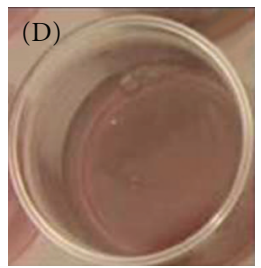

PBS

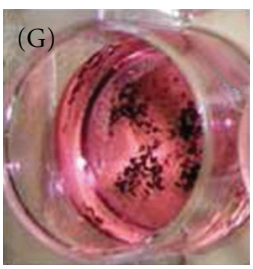

PBS

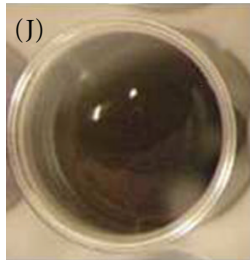

PBS

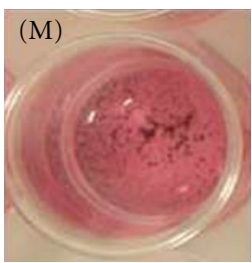

PBS

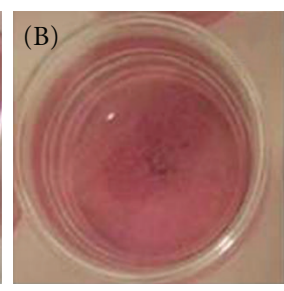

PBS + 1\% DMSO SWCNH-F

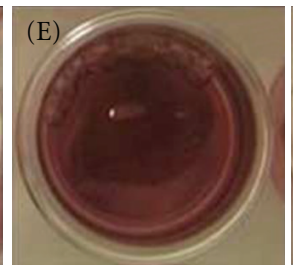

PBS + 1\% DMSO MWCNT

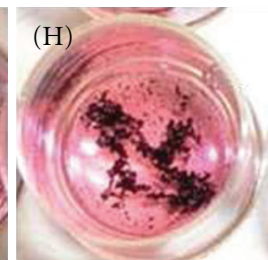

PBS + 1\% DMSO MWCNT-F

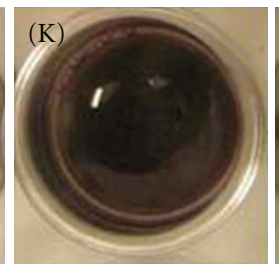

PBS + 1\% DMSO ULSWCNT

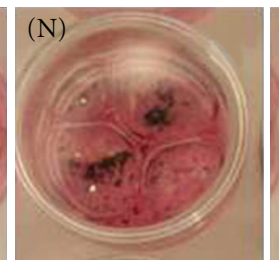

PBS + 1\% DMSO

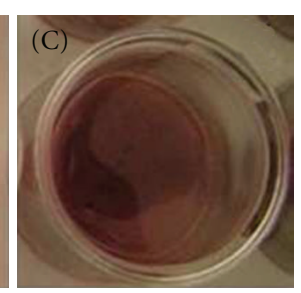

PBS $+1 \%$ Tween 20

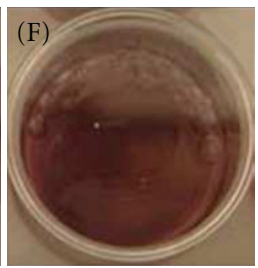

PBS + 1\% Tween 20

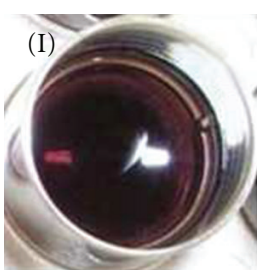

PBS $+1 \%$ Tween 20

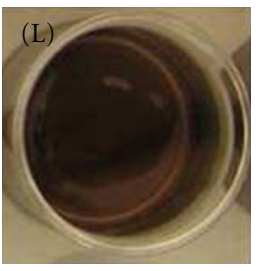

PBS + 1\% Tween 20

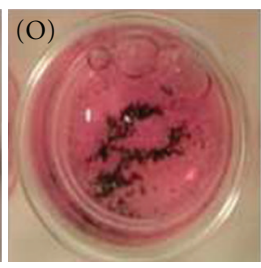

PBS + 1\% Tween 20
FIGURE 11: Photographs of CNTs suspensions using three different liquids $24 \mathrm{~h}$ after dispersion.

MWCNT and SWCNH. It is well known that the surfactants decrease the surface energy of a liquid, allowing easier spreading, and lowering the interfacial tension between two liquids, or between a liquid and a solid. The positive impact of Tween 20 surfactant was observed during dispersion of MWCNTs and SWCNTHs, while in the case of ULSWCNTs, the agglomeration was comparable with samples containing pure PBS and PBS with DMSO (Figure 11(O)). The probable reason could be the length of ULSWCNT, which limits their appropriate dispersion in a solvent. The ULSWCNTs tend to entangle and bundle, which makes their dispersion process and assessment of the surfactant's impact difficult.

A different situation was observed for MWCNT and SWCNH after functionalization in a mixture of acids. Both SWCNH-F and MWCNT-F were well dispersed in all prepared suspensions and maintained their stability up to $24 \mathrm{~h}$ (Figure 11(D, E, F, J, K, and L)). The most effective process was observed for MWCNT-F (Figure 11(J, K, and $\mathrm{L})$ ). The reason for good dispersion of CNTs in pure PBS and PBS with additives was the surface chemistry of these materials. The presence of carboxylic groups on carbon nanotubes changes their wettability from hydrophobic to hydrophilic.

The phagocytosis of CNTs by RAW 264.7 macrophages and its features was qualitatively analysed on the basis of the series of microphotographs gathered in Figures 5, 6, 7, 8, and 9 .

As it results from analyse of these images, the macrophages phagocytose all kinds of CNT with different intensity. The most intensive phagocytosis process was observed for the samples after chemical modification (Figures 6 and 8). The presence of chemical groups on CNTs surface improves their dispersity in solutions. The well-separated carbon nanotubes in a suspension are easier "devoured" by cells. Such images are particularly observed for MWCNTF (Figure 8), where the cells, regardless of types of solvent, were entirely filled with those CNTs. A similar situation was noticed for SWCNH and MWCNT dispersed in PBS containing Tween 20 (Figures 5(c) and 7(c)). In order to compare the phagocytoses effectiveness between MWCNT$\mathrm{F}$ dispersed in different solvent with MWCNT (Figures 8(a), 8(b), and 8(c)) dispersed in PBS containing Tween 20 (Figure $7(\mathrm{c})$ ), the following relationship may be proposed: Figure 8(a) = Figure 8(b) = Figure 8(c) $\geq$ Figure 7(c). Comparable or higher phagocytosis effectiveness observed for MWCNT-F (Figures 8(a), 8(b), and 8(c)) in comparison with MWCNT (Figure 7(c)) can be explained by the presence of shorter carbon nanotubes after their oxidation in an acidic medium.

In the case of samples containing higher size agglomerates, the phagocytosis was distinctly inhibited (Figures 5(a), 5(b), 7(a), 7(b), and 9). Such a case was noticed particularly for the cultures contacted with ULSWCNT where the highest number of cells without carbon nanotubes can be seen (Figure 9). Additionally, the hampered phagocytosis of ULSWCNT by RAW 264.7 could come out of a high length of this form $(15-30 \mu \mathrm{m})$. The influence of the length of CNTs on cell phagocytosis could be confirmed by differentiation between the phagocytosis of SWCNHs (length from 30 to $50 \mathrm{~nm}$ ) or MWCNTs (length between 1-2 $\mu \mathrm{m}$ ) and ULSWCNTs. The influence of the length of the CNTs has already been confirmed in the literature [13]. In spite of the existing agglomerates in SWCNHs and MWCNTsbased suspensions, phagocytosis was easier in comparison to ULSWCNTs-based suspension (Figures 5(a), 5(b), 7(a), 7(b), $9(\mathrm{a})$, and $9(\mathrm{~b}))$. 
The results indicate that the surfactant (Tween 20) has an advantageous influence on the dispersion process of carbon nanotubes in culture media and facilitates phagocytosis by macrophages. The drawback of the application of Tween 20 is its disadvantageous impact on cell morphology (Figure 10). The cell membrane was heterogeneous with numerous vacuoles in cytoplasm (black arrows). The similar results were also observed by Monteiro-Riviere [22]. On the contrary, this phenomenon was not observed for cells harvested with PBS and PBS $+1 \%$ DMSO additions.

\section{Conclusion}

Five kinds of pristine and chemically modified carbon nanotubes were contacted with macrophages to verify their influence on cell phagocytosis. The appropriate dispersion of CNTs in culture media is one of the crucial issues which has a strong impact for interaction cell-CNT. There were used three types of dispersion agents (PBS, PBS + DMSO and PBS + Tween 20). PBS + Tween 20 was the most effective dispersion agent for CNTs, although this surfactant is probably partially toxic for cells, which limits its further application. The use of both pure PBS and PBS with DMSO did not reveal any influence on the good dispersion process of CNT in culture media. The best dispersity was observed for carbon nanotubes after chemical functionalization in an oxidative treatment, irrespective of dispersing agents. Simultaneously, for these kinds of CNTs the highest phagocytosis was observed. The results show that the interaction between CNTs and cells strongly depends on an average agglomerates size and the length of CNTs.

These results provided preliminary information about the influence of different types of CNTs and their degree of dispersity on the phagocytosis processed by macrophage cells. Further investigations are, however, required for better understanding of the mechanism of phagocytosis and the influence of CNTs on the fundamental biological interaction with cells.

\section{Acknowledgment}

This work has been supported by the Marie Curie ActionsIndustry-Academia Partnerships and Pathways (IAPP), FP7PEOPLE-IAPP-2008, project number 230766.

\section{References}

[1] A. M. Schrand, L. Dai, J. J. Schlager, S. M. Hussain, and E. Osawa, "Differential biocompatibility of carbon nanotubes and nanodiamonds," Diamond and Related Materials, vol. 16, no. 12, pp. 2118-2123, 2007.

[2] J. Xu, M. Yudasaka, S. Kouraba, M. Sekido, Y. Yamamoto, and S. Iijima, "Single wall carbon nanohorn as a drug carrier for controlled release," Chemical Physics Letters, vol. 461, no. 4-6, pp. 189-192, 2008.

[3] B. S. Harrison and A. Atala, "Carbon nanotube applications for tissue engineering," Biomaterials, vol. 28, no. 2, pp. 344 353, 2007.
[4] C. P. Firme and P. R. Bandaru, "Toxicity issues in the application of carbon nanotubes to biological systems," Nanomedicine: Nanotechnology, Biology, and Medicine, vol. 6, no. 2, pp. 245-256, 2010.

[5] X. Shi, B. Sitharaman, Q. P. Pham et al., "Fabrication of porous ultra-short single-walled carbon nanotube nanocomposite scaffolds for bone tissue engineering," Biomaterials, vol. 28, no. 28, pp. 4078-4090, 2007.

[6] K. Matsumoto, C. Sato, Y. Naka, A. Kitazawa, R. L. D. Whitby, and N. Shimizu, "Neurite outgrowths of neurons with neurotrophin-coated carbon nanotubes," Journal of Bioscience and Bioengineering, vol. 103, no. 3, pp. 216-220, 2007.

[7] A. Fraczek, E. Menaszek, C. Paluszkiewicz, and M. Blazewicz, "Comparative in vivo biocompatibility study of single- and multi-wall carbon nanotubes," Acta Biomaterialia, vol. 4, no. 6, pp. 1593-1602, 2008.

[8] C. A. Poland, R. Duffin, I. Kinloch et al., "Carbon nanotubes introduced into the abdominal cavity of mice show asbestoslike pathogenicity in a pilot study," Nature Nanotechnology, vol. 3, no. 7, pp. 423-428, 2008.

[9] A. A. Shvedova, V. Castranova, E. R. Kisin et al., "Exposure to carbon nanotube material: assessment of nanotube cytotoxicity using human keratinocyte cells," Journal of Toxicology and Environmental Health Part A, vol. 66, no. 20, pp. 1909-1926, 2003.

[10] D. B. Warheit, B. R. Laurence, K. L. Reed, D. H. Roach, G. A. M. Reynolds, and T. R. Webb, "Comparative pulmonary toxicity assessment of single-wall carbon nanotubes in rats," Toxicological Sciences, vol. 77, no. 1, pp. 117-125, 2004.

[11] V. E. Kagan, H. Bayir, and A. A. Shvedova, "Nanomedicine and nanotoxicology: two sides of the same coin," Nanomedicine: Nanotechnology, Biology, and Medicine, vol. 1, no. 4, pp. 313316, 2005.

[12] A. Bianco, K. Kostarelos, C. D. Partidos, and M. Prato, "Biomedical applications of functionalised carbon nanotubes," Chemical Communications, no. 5, pp. 571-577, 2005.

[13] Y. Sato, A. Yokoyama, K. I. Shibata et al., "Influence of length on cytotoxicity of multi-walled carbon nanotubes against human acute monocytic leukemia cell line THP-1 in vitro and subcutaneous tissue of rats in vivo," Molecular BioSystems, vol. 1, no. 2, pp. 176-182, 2005.

[14] P. Wick, P. Manser, L. K. Limbach et al., "The degree and kind of agglomeration affect carbon nanotube cytotoxicity," Toxicology Letters, vol. 168, no. 2, pp. 121-131, 2007.

[15] K. L. Aillon, Y. Xie, N. El-Gendy, C. J. Berkland, and M. L. Forrest, "Effects of nanomaterial physicochemical properties on in vivo toxicity," Advanced Drug Delivery Reviews, vol. 61, no. 6, pp. 457-466, 2009.

[16] P. M. V. Raja, J. Connolley, G. P. Ganesan et al., "Impact of carbon nanotube exposure, dosage and aggregation on smooth muscle cells," Toxicology Letters, vol. 169, no. 1, pp. 51-63, 2007.

[17] P. Cherukuri, S. M. Bachilo, S. H. Litovsky, and R. B. Weisman, "Near-infrared fluorescence microscopy of singlewalled carbon nanotubes in phagocytic cells," Journal of the American Chemical Society, vol. 126, no. 48, pp. 15638-15639, 2004.

[18] R. Singh, D. Pantarotto, D. McCarthy et al., "Binding and condensation of plasmid DNA onto functionalized carbon nanotubes: toward the construction of nanotube-based gene delivery vectors," Journal of the American Chemical Society, vol. 127, no. 12, pp. 4388-4396, 2005. 
[19] K. Ajima, M. Yudasaka, T. Murakami, A. Maigné, K. Shiba, and S. Iijima, "Carbon nanohorns as anticancer drug carriers," Molecular Pharmaceutics, vol. 2, no. 6, pp. 475-480, 2005.

[20] M. Bottini, S. Bruckner, K. Nika et al., "Multi-walled carbon nanotubes induce T lymphocyte apoptosis," Toxicology Letters, vol. 160, no. 2, pp. 121-126, 2006.

[21] R. Rastogi, R. Kaushal, S. K. Tripathi, A. L. Sharma, I. Kaur, and L. M. Bharadwaj, "Comparative study of carbon nanotube dispersion using surfactants," Journal of Colloid and Interface Science, vol. 328, no. 2, pp. 421-428, 2008.

[22] N. A. Monteiro-Riviere, A. O. Inman, Y. Y. Wang, and R. J. Nemanich, "Surfactant effects on carbon nanotube interactions with human keratinocytes," Nanomedicine: Nanotechnology, Biology, and Medicine, vol. 1, no. 4, pp. 293-299, 2005.

[23] S. H. Su, W. T. Chiang, C. C. Lin, and M. Yokoyama, "Multiwall carbon nanotubes: purification, morphology and field emission performance," Physica E, vol. 40, no. 7, pp. 23222326, 2008.

[24] V. Datsyuk, M. Kalyva, K. Papagelis et al., "Chemical oxidation of multiwalled carbon nanotubes," Carbon, vol. 46, no. 6, pp. 833-840, 2008.

[25] J. Liu, A. G. Rinzler, H. Dai et al., "Fullerene pipes," Science, vol. 280, no. 5367, pp. 1253-1256, 1998.

[26] E. B. Barros, A. G. S. Filho, V. Lemos et al., "Charge transfer effects in acid treated single-wall carbon nanotubes," Carbon, vol. 43, no. 12, pp. 2495-2500, 2005.

[27] R. Tucknott and S. N. Yaliraki, "Aggregation properties of carbon nanotubes at interfaces," Chemical Physics, vol. 281, no. 2-3, pp. 455-463, 2002. 

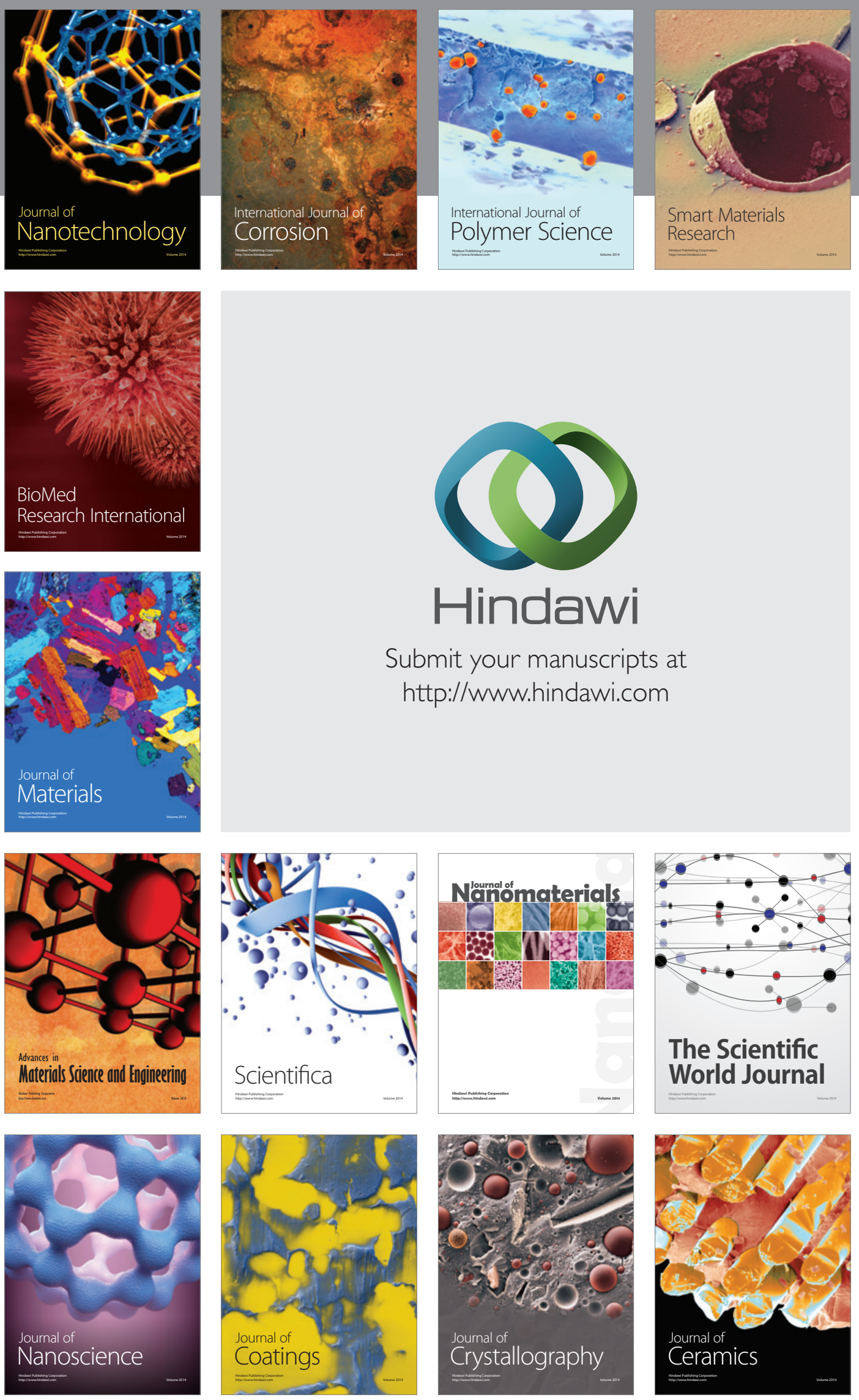

The Scientific World Journal

Submit your manuscripts at

http://www.hindawi.com

\section{World Journal}

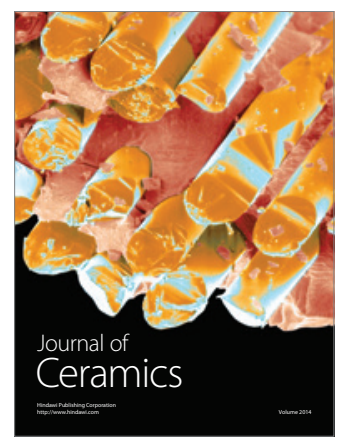

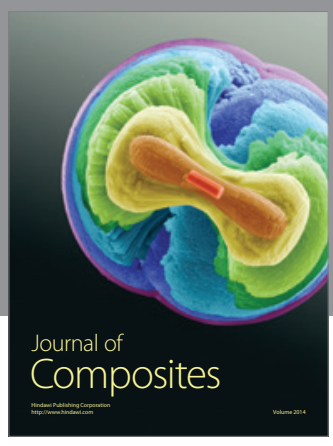
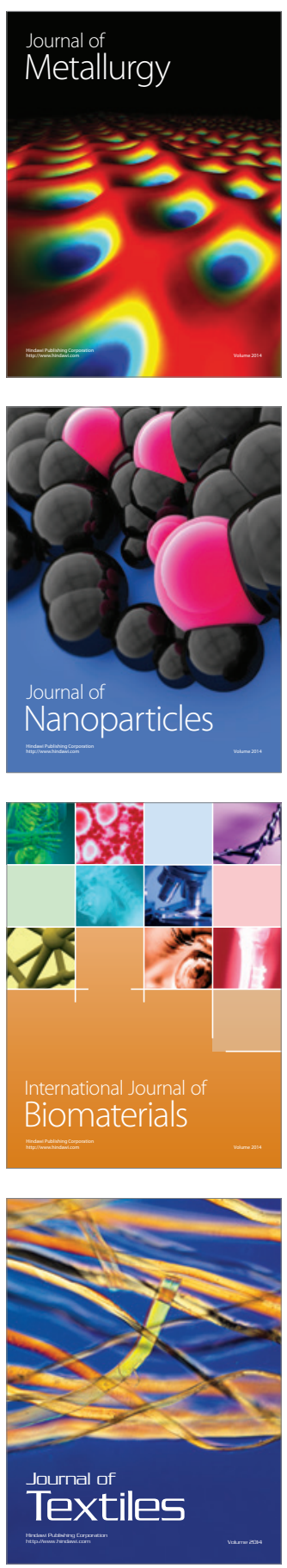